\title{
Identification of Vehicle Suspension Shock Absorber Squeak and Rattle Noise Based on Wavelet Packet Transforms and a Genetic Algorithm- \\ Support Vector Machine
}

\author{
Hai B. Huang ${ }^{a, b}$, Ren X. Li ${ }^{\text {a }}$, Xiao R. Huang ${ }^{\text {a }}$, Teik C. Lim ${ }^{b}$, Wei P. Ding ${ }^{a}$,* \\ ${ }^{a}$ Institute of Automotive Engineering Research, Southwest Jiaotong University, 610031, Cheng Du, Si \\ Chuan, China \\ ${ }^{b}$ Vibro-Acoustics and Sound Quality Research Laboratory, College of Engineering and Applied \\ Science, University of Cincinnati, 45221 Cincinnati, Ohio, USA \\ *Corresponding author, Tel.: (+28)87600695 \\ E-mail: huanghaibo214@my.swjtu.edu.cn (H.B. Huang), rxli@home.swjtu.edu.cn (R.X. Li), \\ xiaoronghuang@my.swjtu.edu.cn (X.R. Huang), limt@ucmail.uc.edu (T. C. Lim), \\ dwp@home.swjtu.edu.cn (W.P. Ding).
}

Abstract: The squeak and rattle (S\&R) noise of a vehicle's suspension shock absorber substantially influences the psychological and physiological perception of passengers. In this paper, a state-of-the-art method, specifically, a genetic algorithm-optimized support vector machine (GA-SVM), which can select the most effective feature subsets and optimize the model's free parameters, is proposed to identify this specific noise. A vehicular road test and a shock absorber rig test are conducted to investigate the relationship between these features, and then an approach for quantifying the shock absorber S\&R noise is given. Pre-processed signals are decomposed through a wavelet packet transform (WPT), and two criteria, namely, the wavelet packet energy (WPE) and wavelet packet sample entropy (WPSE), are introduced as the feature extraction methods. Then, the two extracted feature sets are compared based on this genetic algorithm. Another advanced method, known as the genetic algorithm-optimized back propagation neural network (GA-BPNN), is introduced for comparison to illustrate the superiority of the newly developed GA-SVM model. The result shows that the WPSE can extract more useful features than the WPE and that the GA-SVM is more effective and 
efficient than the GA-BPNN. The proposed approach could be retrained and extended to address other fault identification problems.

Key words: Squeak and rattle (S\&R); Wavelet packet transform (WPT); Wavelet packet energy (WPE); Wavelet packet sample entropy (WPSE); Genetic algorithm (GA); Support vector machine (SVM)

\section{Introduction}

Vehicle noise, vibration and harshness $(\mathrm{NVH})$ is very important to a customer's perception of vehicle quality. Therefore, much effort is invested in improving car riding comfort and interior sound quality [1]. However, as the main sound and vibration sources have been increasingly well controlled, other types of noise from previously ignored components, such as gear noise [2], heating, ventilation, and air conditioning (HVAC) noise [3], and wiper noise [4], have become prevalent. Among these numerous component noises, suspension shock absorber squeak and rattle $(\mathrm{S} \& \mathrm{R})$ noise has been subject to increasing awareness from vehicular passengers.

The suspension shock absorber, which serves as the primarily stressed component, inevitably leads to shock and vibration and thus causes intensive noise during functioning. The so-called suspension shock absorber $\mathrm{S} \& \mathrm{R}$ noise is a structure-borne sound that is induced by the suspension shock absorber acting on the car body [5,6]. Specifically, when the internal components of the suspension shock absorber are resonating, the road excitation force is magnified and acts on the car body through the piston rod system of the shock absorber, which creates S\&R noise [7]. This distinctive sound worsens when the car is driving on a bumpy road [8]. Identifying a vehicle's suspension shock absorber S\&R noise is essential and realistic for the following three reasons. First, S\&R noise weakens the interior sound quality of the vehicle and negatively affects the passengers' psychology and physiology. Second, this specific noise can worsen an automobile brand's image, increase the return rate, and raise the costs of vehicle and component companies. Third, although the structural design of the suspension shock absorber is satisfied, biases and inconsistencies still exist in manufacturing; as a result, some mass-produced shock absorbers can induce S\&R noise.

The identification of vehicular suspension shock absorber S\&R noise formerly depended on the 
subjective evaluation of a vehicular road test because this noise can be identified within the limits of human hearing perception. Some vehicular road studies found that S\&R noise is related to the vibration characteristics of the car body at the locations where shock absorbers are assembled $[9,10]$. However, conducting a large number of vehicular road tests to identify S\&R noise requires cooperation between motor companies and the automobile parts companies, which largely increases the manpower and cost of the car. Conversely, a rig test for suspension shock absorber S\&R noise can overcome the disadvantages of requisite vehicular road tests. In one study, a quarter-car suspension system was used to investigate the $\mathrm{S} \& \mathrm{R}$ noise of a shock absorber and found that powerful impact acceleration along a shock absorber resulted in an intensive vibration of the car body, which can create a high S\&R noise level [11]. Meanwhile, reports in the literature $[8,12]$ have shown similar results. The methods used to extract the S\&R characteristics and establish the relationships between the obtained features and human hearing perception are key factors in the identification of S\&R noise. Although some researchers and engineers have engaged in developing means of identifying the $S \& R$ noise problem [13], few methods have been generally applied in industry. Therefore, a practical and state-of-the-art method for shock absorber S\&R noise identification on a test rig, that also has a high correlation relationship with the subjective evaluation in a vehicular road test, is urgently needed in automobile engineering.

Extracting the S\&R characteristics is essential to identify the precise identification of the associated S\&R noise of a shock absorber. Some advanced time-frequency analysis techniques, such as the short time Fourier transform (STFT), Wigner-Ville distribution (WVD), empirical mode decomposition (EMD), wavelet transform (WT) and wavelet packet transform (WPT), have been widely used in sound and vibration signal processing to extract features from signals. Each of these methods has its own advantages and drawbacks. Compared to the STFT, the WT and WPT can process a signal using a variable resolution window rather than a fixed resolution window, but a wavelet function should be pre-defined [14]. The WVD is a special type of bilinear time-frequency transform that can provide the instantaneous power spectrum density of a signal, but the cross-terms will seriously affect its performance; therefore, the pseudo Wigner-Ville distribution (PWVD) and the 
smoothed pseudo Wigner-Ville distribution (SPWVD) have been proposed to suppress the influence of these cross-terms [15]. EMD is the kernel algorithm of the Hilbert-Huang transform (HHT), which can adaptively decompose a signal to several intrinsic mode functions (IMF) according to its own characteristics, but the decomposed IMF results in frequency overlap and is easily influenced by noise; hence, the ensemble empirical mode decomposition (EEMD) approach has been developed to solve these problems [16]. However, in practical use, few signal processing methods perform overwhelmingly better than others. Therefore, the method that should be selected mainly depends on the characteristics of an original signal and the purpose of the analysis.

Intelligent recognition methods should be considered to simulate the human auditory system because of the complexity of the human perception process. The artificial neural network (ANN), support vector machine (SVM) and multiple linear regression (MLR) approaches have been frequently mentioned in the literature $[17,18]$. Based on extensive empirical research, the ANN and SVM techniques have proven to be very powerful tools to map the nonlinear characteristics between the input features and the output targets, compared to the traditional MLR method [18]. The general parameters and hyper parameters of those models should be carefully selected to obtain satisfactory performance. However, no appropriate theoretical method exists to guide parameter selection. An advanced intelligent optimization algorithm should be introduced because of the irregularity of the parameter selection process. The genetic algorithm (GA), particle swarm optimization (PSO) and simulated annealing algorithm (SAA) are excellent methods to solve the nonlinear and multi-extremum programming in a broad range of research fields $[19,20]$. Therefore, a combination of an intelligent recognition method and an intelligent optimization algorithm should adequately quantify the S\&R noise level of a vehicle's suspension shock absorber.

From the above descriptions, the pattern identification methods show a trend in development from traditional to intelligent approaches. In particular, the methods for S\&R noise feature extraction and S\&R noise pattern recognition must be carefully designed to identify shock absorber S\&R noise. In this work, a new identification approach that combines the WPT and GA optimized SVM (GA-SVM) is 
proposed to detect the $\mathrm{S} \& \mathrm{R}$ noise of a vehicle's suspension shock absorber and is validated based on a vehicular road test and shock absorber test rig. The scheme of this research is presented in Fig. $\mathbf{1}$.

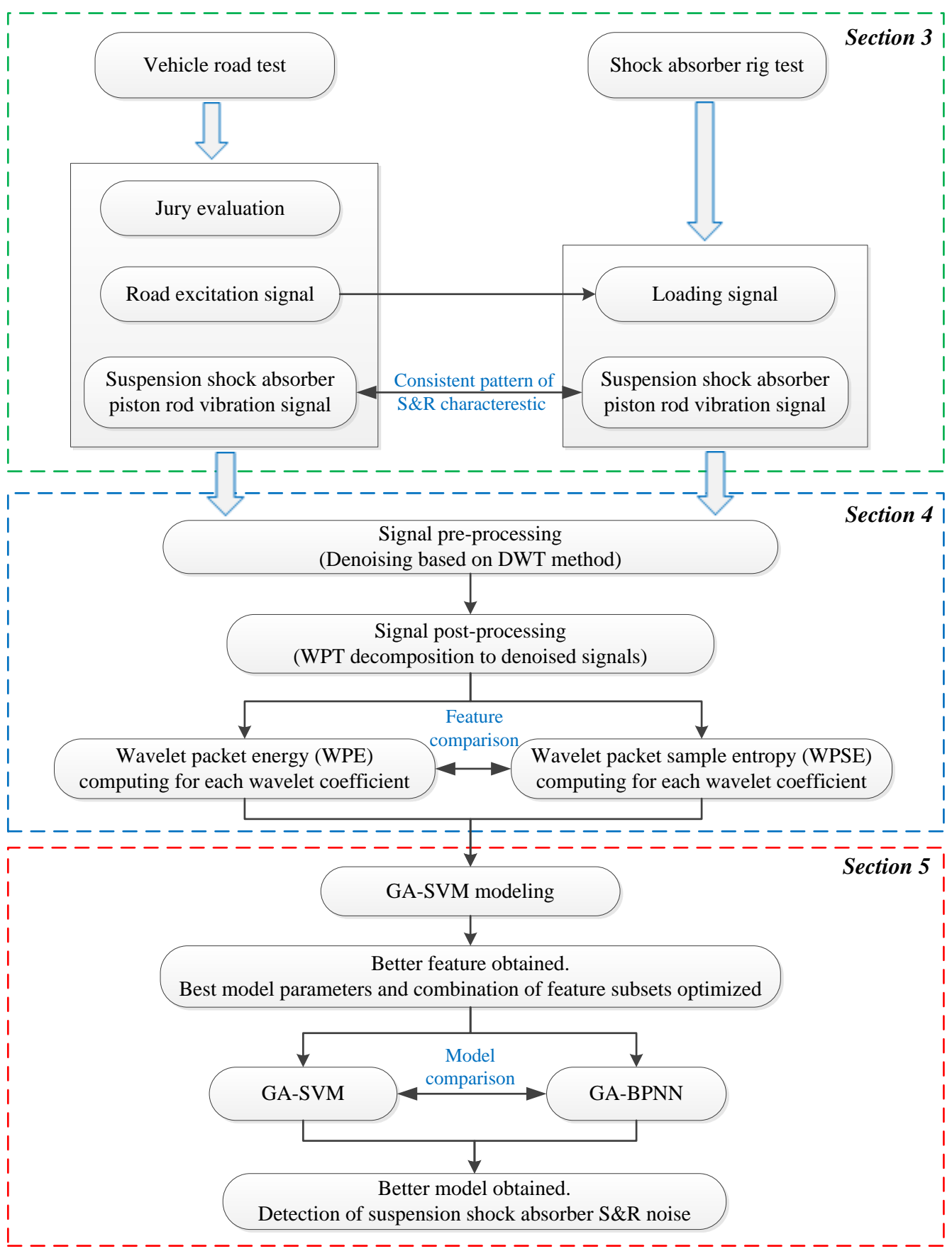

Fig. 1. Identification scheme of the vehicular suspension shock absorber S\&R noise

\section{Theory background}

\subsection{Wavelet packet transform}

Compared with the STFT, the WT can provide a varying time and frequency resolution when used in signal processing. However, this resolution is an equal exponential frequency partition [21] and 
decreases with increasing frequency. This shortcoming can sometimes reduce the accuracy when analyzing strong nonstationary or transient signals. The WPT is proposed to acquire more useful information. The WPT can offer a more detailed and adjustable resolution according to the characteristics and requirements of the signal [22]. Similar to the WT, the WPT can decompose a signal into an approximation coefficient and a detail coefficient. However, in contrast to the WT, the WPT can then split the detail coefficient into another approximation coefficient and another detail coefficient. An example of a 4-level decomposition tree for the WT and WPT is presented in Fig. 2, where $x$ is the original signal, $\mathrm{L}$ is a low-pass filter, and $\mathrm{H}$ is a high-pass filter.

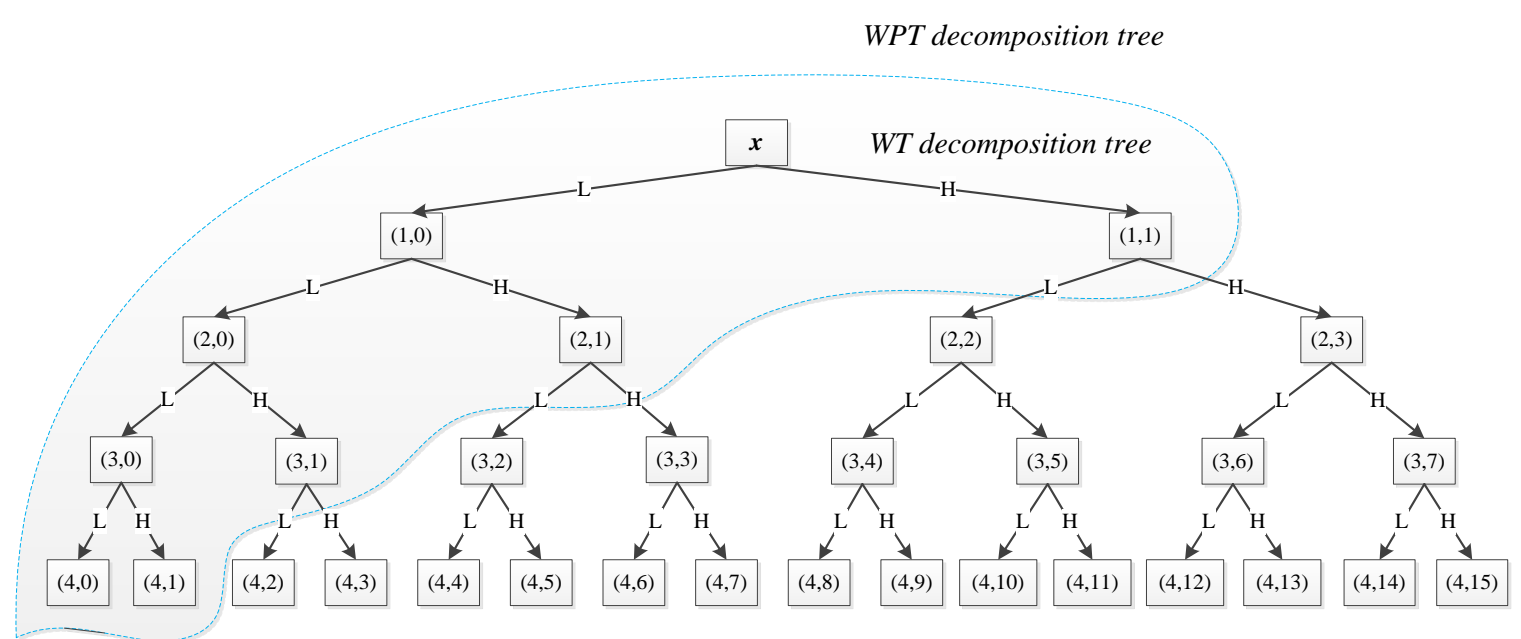

Fig. 2. Decomposition tree for WT and WPT

Theoretically, given the scaling function $\varphi(t)$ and the wavelet function $\psi(t)$, which are defined as $u_{0}(t)=\varphi(t)$ and $u_{1}(t)=\psi(t)$, the recursion equation of a wavelet packet is derived as follows:

$$
\left\{\begin{array}{l}
u_{2 n}(t)=\sqrt{2} \sum_{k} h_{k} u_{n}(2 t-k) \\
u_{2 n+1}(t)=\sqrt{2} \sum_{k} g_{k} u_{n}(2 t-k)
\end{array}\right.
$$

where $\square_{\square}$ and $\square_{\square}$ are the coefficients of the low-pass filter and the high-pass filter, respectively. Given an original signal $x(t)$ as $C^{0,0}$, the decomposed approximation coefficient and detail coefficient of the WPT can be obtained by

$$
\left\{\begin{array}{l}
C_{m}^{j, 2 n}=\sum_{k=-\infty}^{\infty} h_{2 m-k}^{*} C_{k}^{j+1, n} \\
C_{m}^{j, 2 n+1}=\sum_{k=-\infty}^{\infty} g_{2 m-k}^{*} C_{k}^{j+1, n}
\end{array}\right.
$$


where $j, m$ and $n$ are the scale, translation and oscillation parameters, respectively. Thus, the completed WPT can be achieved via Eq. (3).

Meanwhile, the reconstruction of the WPT coefficients is defined as

$$
C_{m}^{j+1, n}=\sum_{k=-\infty}^{\infty} h_{2 m-k} C_{k}^{j, 2 n}+\sum_{k=-\infty}^{\infty} g_{2 m-k} C_{k}^{j, 2 n+1}
$$

2.2 Genetic algorithm-support vector machine

\subsubsection{Support vector machine}

SVMs were first proposed by Vapnik in 1995 [23] and have been demonstrated to be a very powerful tool for solving regression, classification and pattern recognition problems. The SVM classifier was originally developed for 2-class classifications. Subsequently, some researchers have improved the algorithm and have applied it to $\mathrm{N}$-class $(\mathrm{N}>2)$ classification problems [24]. The basic idea of the SVM classifier is to map the input data from a low-dimensional feature space to a high-dimensional feature space using nonlinear mapping and then performing linear classification in this new space. The topological structure of SVMs is shown in Fig. 3.

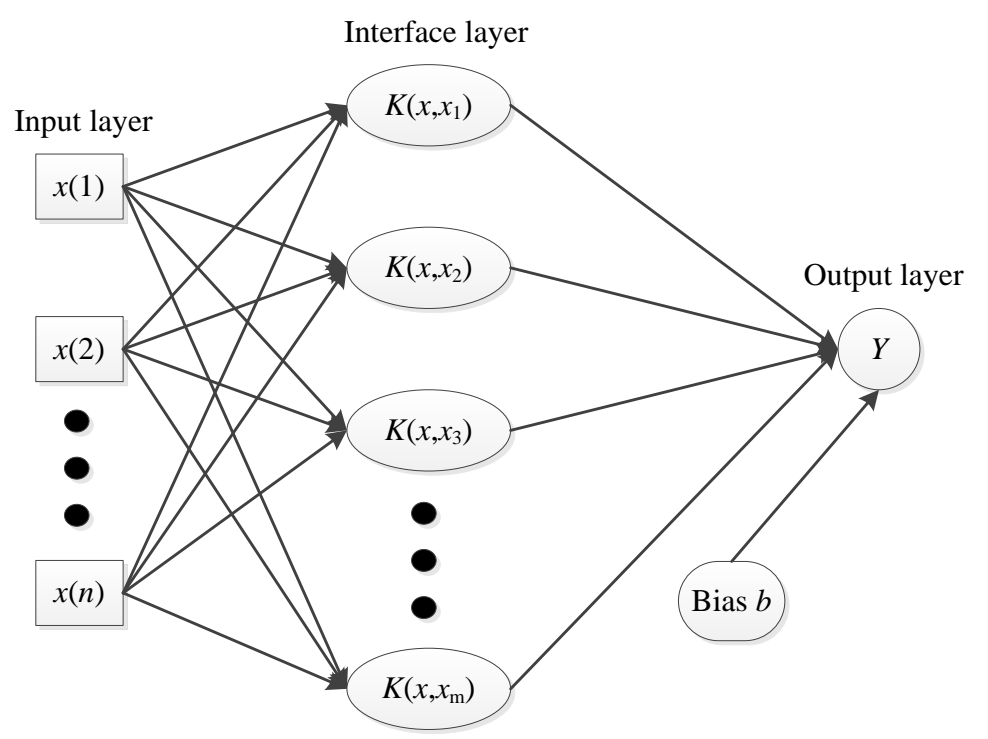

Fig. 3. Topological structure of the SVM, where $n$ is the dimension of the input data, $K$ is the kernel function and $m$ is the number of support vectors

Given a dataset $\left\{x_{i}, y_{i} \mid i=1,2, \ldots, N\right\}, x_{i} \in \mathrm{R}^{d}$ represents the independent variables, $y_{i} \in$ $\{+1,-1\}$ represents the dependent variables, and $N$ represents the number of training samples. When 
the two classes can be linearly separable, one can determine a hyperplane $f(x)=0$ that separates the given data, as shown in Eq. (4)

$$
f(x)=\sum_{i=1}^{N} w \cdot x_{i}+b=0
$$

where $w$ is the weight vector, and $b$ is the bias term. These terms are used to define the position of the separating hyperplane, which should satisfy the following constraints:

$$
y_{i}\left(w \cdot x_{i}+b\right) \geq 1, i=1,2, \ldots, N
$$

Positive slack variables $\xi_{i}$ have been introduced to measure the distance between the margin and vectors $x_{i}$ that lie on the wrong side of the hyperplane. Then, the optimal separating hyperplane can be obtained via the following optimization problem:

$$
\begin{array}{ll}
\min & \frac{1}{2}\|w\|^{2}+C \sum_{i=1}^{N} \xi_{i}, \quad i=1,2, \ldots, N \\
\text { s.t. } & \left\{\begin{array}{l}
y_{i}\left(w \cdot x_{i}+b\right) \geq 1-\xi_{i} \\
\xi_{i} \geq 0
\end{array}\right.
\end{array}
$$

where $C$ is the soft margin parameter.

By introducing the nonnegative Lagrange multiplier $\alpha_{i}$, Eq. (6) can be transformed into the dual quadratic optimization problem, as presented in Eq. (7)

$$
\begin{array}{ll}
\max & L(\alpha)=\sum_{i=1}^{N} \alpha_{i}-\frac{1}{2} \sum_{i, j=1}^{N} \alpha_{i} \alpha_{j} y_{i} y_{j} K\left(x_{i}, x_{j}\right) \\
\text { s.t. } & \sum_{i=1}^{N} \alpha_{i} y_{j}=0,0 \leq \alpha_{i} \leq C, i=1,2, \ldots, N
\end{array}
$$

where $K\left(x, x_{i}\right)$ is a kernel function. For the linear classification problem, $K\left(x, x_{i}\right)=x \cdot x_{i}$. Training samples with nonzero weight $\alpha_{i}$ are called support vectors. Thus, the decision function of the SVM is defined as follows:

$$
f(x)=\operatorname{sign}\left(\sum_{i=1}^{N} \alpha_{i} y_{i} K\left(x, x_{i}\right)+b\right)
$$

The elegance of using a kernel function lies in the fact that the kernel function replaces the inner product in the high-dimension feature space, which significantly reduces the number of calculations and the complexity. Thinking of the kernel, $K\left(x, x_{i}\right)$, as comparing patterns or evaluating the 
proximity of objects in their feature space may be useful [25]. Typical examples of kernel functions are linear, polynomial, Gaussian radial basis and sigmoid functions. Each kernel function has unique characteristics. The Gaussian radial basis function kernel (RBF) is the most commonly used in function estimation problems because of its effectiveness and robustness. The form of the RBF is defined as follows:

$$
K\left(x, x_{i}\right)=\exp \left(-\gamma\left\|x-x_{i}\right\|^{2}\right)
$$

where $\gamma=1 / 2 \sigma^{2}$ is the kernel parameter, in which $\sigma$ is the width parameter of the RBF.

SVMs have limitations in feature subset selection and parameter optimization. Feature subset selection is essentially an optimization process that involves searching the feature space for several features that are nearly optimal with respect to some accuracy value [26]. The importance of this process rises with an increasing number of input feature attributes. Feature subset selection is used for three reasons: (1) the simplification of models to make them easier to interpret by researchers, (2) shorter training times, and (3) the enhancement of the generalization by reducing overfitting. Additionally, the performance of SVMs depends on many factors, including the soft margin parameter $C$, the type of kernel function, and its corresponding parameters. Selecting an appropriate combination of these parameters plays an important role in the SVM recognition process, but optimizing the parameters for the best prediction performance is still a crucial problem. Therefore, we propose a genetic algorithm (GA) in this study as the method to solve these two problems.

\subsubsection{Genetic algorithm-optimized SVM}

A GA is a global optimal algorithm based on the theory of natural selection and evolution. GAs use the principle of 'survival of the fittest' to progressively accept better solutions for problems. These algorithms are inspired by and named after biological processes of inheritance, natural selection, genetic crossover and genetic mutation [27]. A GA begins with a set of solutions that are represented by initialized chromosomes, which subsequently evolve and update to new chromosomes, called offspring, via genetic operators. The genetic operator is the key procedure in GAs and can be implemented in three steps: (1) Selection. Some excellent chromosomes are selected and reproduced 
based on the fitness function, that is, chromosomes with higher fitness values are more likely to be selected and to yield offspring in the next generation. (2) Crossover. Genes (binary code or others) between two chromosomes are randomly exchanged by means of the $N$-point $(N=1,2, \ldots)$ crossover principle. (3) Mutation. Genes of the chromosomes would have the chance to alter their states from 0 to 1 , or 1 to 0 , but the probability is very low. After the genetic operator process, the offspring substitutes for the old population and forms a new population in the next generation. The evolutionary procedure iterates until the terminal condition is achieved.

A GA is introduced to improve the performance of the SVM model and reduce the redundancy of the input features. We first obtain the dataset, and the kernel function of the SVM should be decided because it will affect the sample distribution in the mapping space. Second, the GA is applied to encode the SVM parameters and the input features, which are represented by a chromosome that is composed of encoding values. The length of the bit string that represents the SVM parameters depends on the required calculation precision. The length of the bit string that represents the input feature equals the dimension of the features, and each bit of the chromosome determines whether the corresponding feature is selected. Specifically, 1 or 0 in each bit means the corresponding feature is either selected as an input feature or is not. The initial chromosomes of the population are randomly generated. Third, we apply the SVM recognition accuracy as the fitness function and implement the genetic operator until the terminal condition is satisfied. The scheme of the GA-optimized SVM procedure is shown in Fig. 4. 


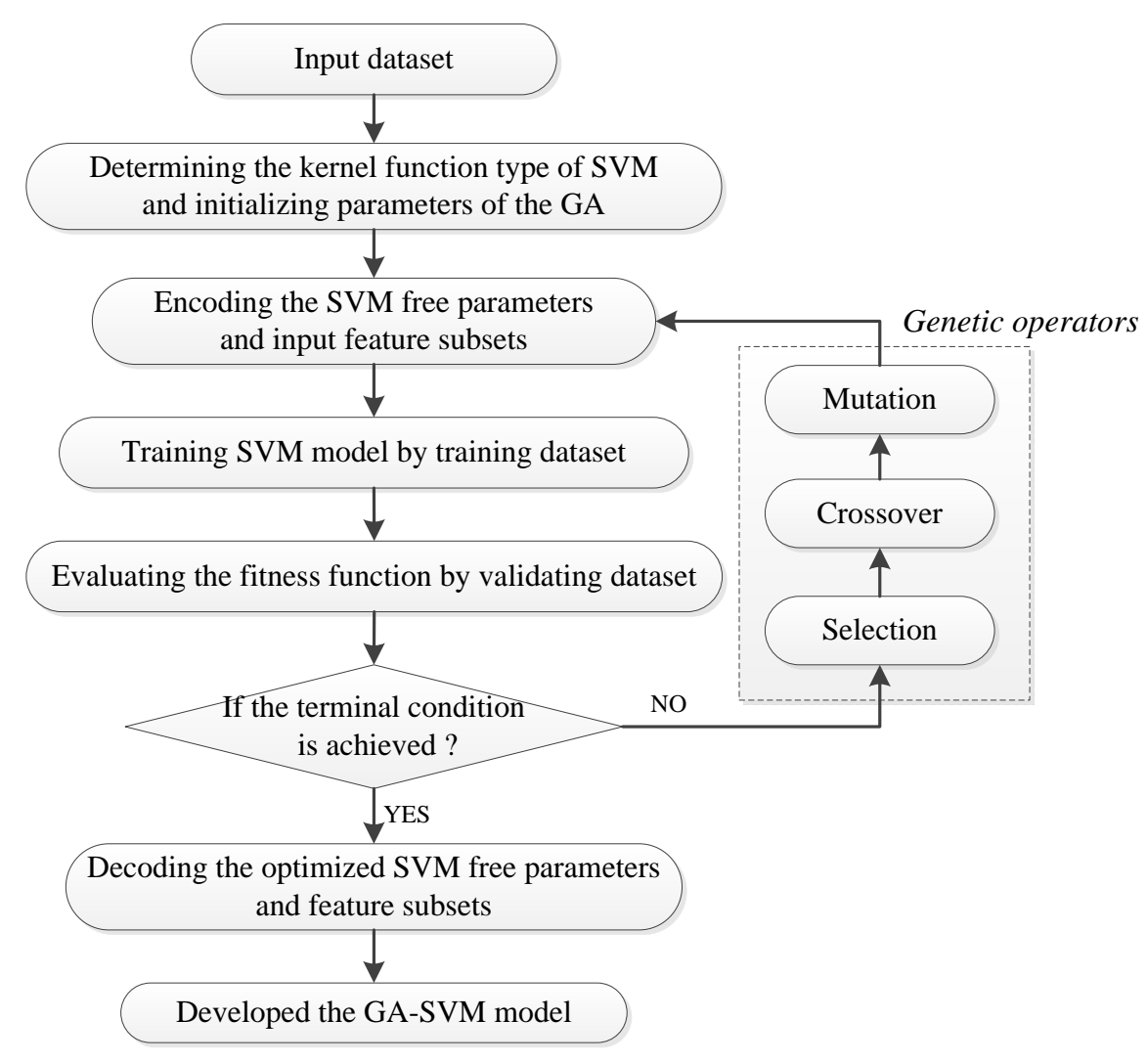

Fig. 4. Overall procedure of the GA-SVM

\section{Vehicular road test and shock absorber rig test}

To identify the $S \& R$ noise of vehicle suspension shock absorbers, it is essential to know how to measure this specific sound or how to quantify this sound in an alternative way. In this section, the $\mathrm{S} \& \mathrm{R}$ noise is examined through a vehicular road test and shock absorber test rig, separately, to illustrate the relationship between S\&R noise and the vibration characteristic of shock absorbers, and then a quantification method for measuring the $S \& R$ noise is given.

3.1 Vehicular road test for suspension shock absorber S\&R noise

\subsubsection{Signal acquisition}

Suspension shock absorber S\&R noise is not loud compared to general interior noise; however, this particular noise has been found to have a subtle influence on passengers' emotions, especially on rough roads. Therefore, the engine of the test vehicle was shut down, and the test vehicle was allowed to coast on a rough test road with a gradient of approximately $8 \%$ at a speed of approximately $15 \mathrm{~km} / \mathrm{h}$ (by the driver) to control the interior noise and maintain the passengers' focus on the suspension shock absorber S\&R noise. No sound-reflecting objects were present within $20 \mathrm{~m}$ in the area around the test 
road. Because the vehicle's engine and the power transmission system were not operating, the suspension shock absorber S\&R noise was predominant and was reflected in the test vehicle's interior noise. One hundred and fifty return-to-company suspension shock absorbers with different S\&R noise levels were used as the test samples in this work. In one experiment, only one suspension shock absorber was replaced by the sample shock absorber, while the remaining three were normal. The interior noise was recorded using microphones above the driver seat with a signal length of $10 \mathrm{~s}$ and a sampling rate of $44.1 \mathrm{kHz}$ according to the vehicle interior noise measurement method that was introduced by the standard GB/T18697 [28]. The vibration acceleration of the piston rod and the bottom of the suspension shock absorber were measured simultaneously in the test. The former vibration signal was recorded to measure the shock effect of the suspension shock absorber that was acting on the car body, and the latter vibration signal was used to simulate the road excitation as the loading signal for the test rig. A signal length of $10 \mathrm{~s}$ and an experimental sampling rate of $2.5 \mathrm{kHz}$ were adopted for the vibration signals. Fig. 5 schematically illustrates the vehicular road test procedure. The recording test was performed once for each point and each shock absorber sample considering the collection positions for the interior noise and vibration signals. All the recorded signals were saved on a mobile workstation for subsequent analysis. 

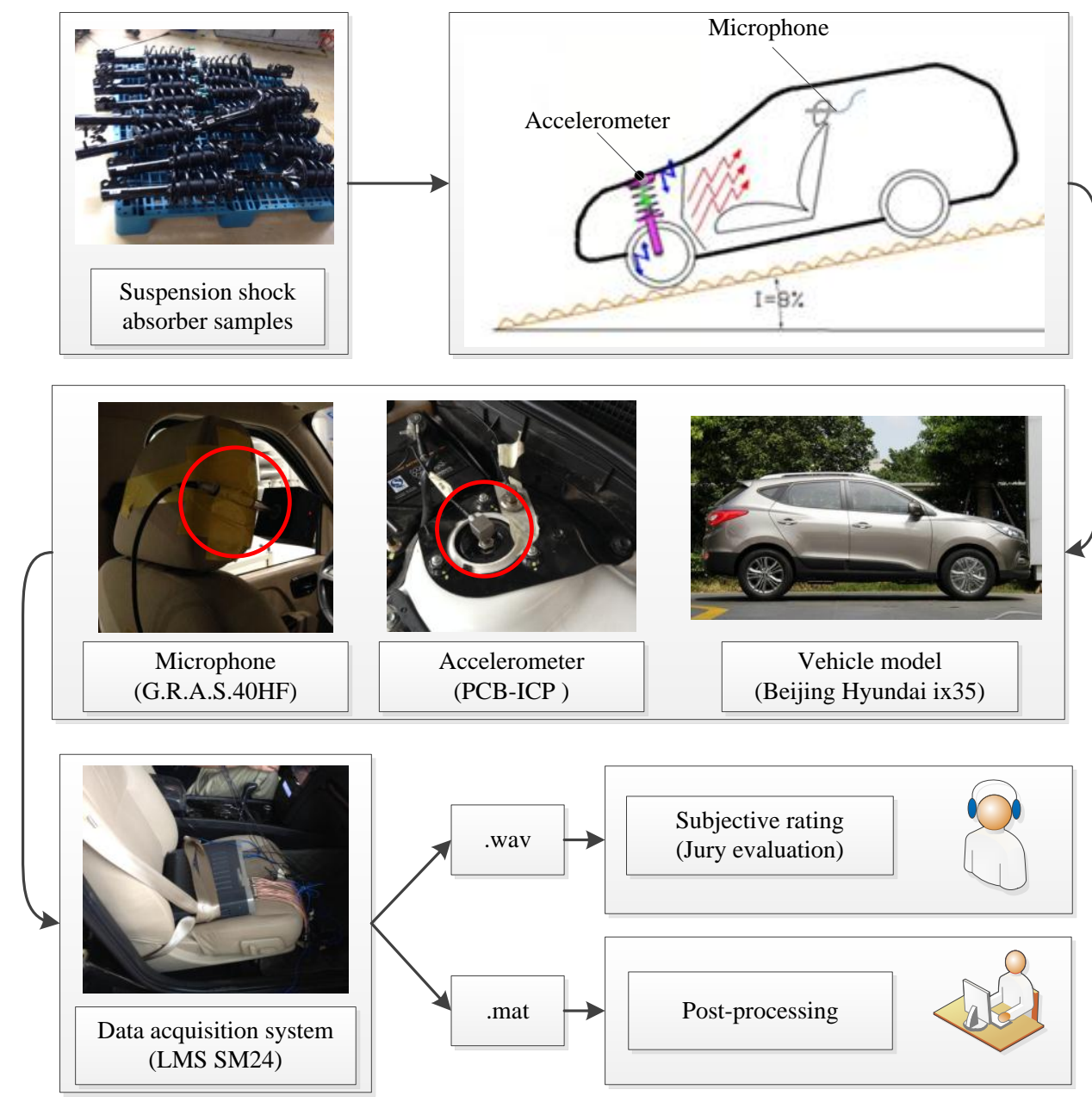

Fig. 5. Vehicular road test process for the identification of suspension shock absorber S\&R noise

\subsubsection{Collected data pre-processing}

The measured noise and vibration signals from the vehicular road test contained noise; therefore, these signals must be denoised to avoid signal distortion by certain additive interference, which may be introduced by the hardware of the test system and other noises from external conditions. The wavelet denoising method has proven to be an effective noise reduction method, and some of its properties are highly advantageous relative to those of a conventional filter [29]. Therefore, a discrete wavelet transform (DWT) denoising method [30] was adopted in this study for the non-stationary signals. Parameters of the DWT denoising were selected as followed: Daubechies wavelet " $d b 5$ " as a wavelet function, 4 levels of decomposition, and a soft threshold for denoising. Using the DWT denoising technique, signal values that were less than the determinate threshold are set to zero, and others remain as the original values. 


\subsubsection{Jury evaluation}

A jury test was conducted using a semantic rating method [31] to evaluate the suspension shock absorber S\&R noise levels. Thirty reviewers (18 males and 12 females between 20 and 50 years of age) with normal hearing and university or automobile company affiliations participated in the subjective evaluation, and all the participants were screened to judge whether they were fit for this study. The recorded interior noise signals were pre-processed using the CoolEdit software to restrict each sound to $5 \mathrm{~s}$ to reduce the total estimation time and improve the evaluation accuracy rate. The processed sound samples were randomly ordered, and the participants rated the levels of the suspension shock absorber S\&R noise on a scale from 1 to 5 , as shown in Tab. 1. The subjective evaluation condition was a quiet and ventilated laboratory, and high-precision Sennheiser HD800 headphones were used for the jury evaluation. Before the formal test, participants could randomly select noise samples to prepare themselves for the overall S\&R noise situation.

Fig. 6 shows the subjective ratings of the $S \& R$ grade of the measured sounds, where the $X$-axis is the test number of the suspension shock absorber sample, and the Y-axis is the average subjective rating of the S\&R noises. As seen in Fig. 6, the jury evaluation was relatively stable and can thus be used in the subsequent study. Rounded values of the subjective ratings were used for identification to clearly classify the shock absorber S\&R grades.

Tab. 1 Definitions of suspension shock absorber S\&R noise during the jury evaluation

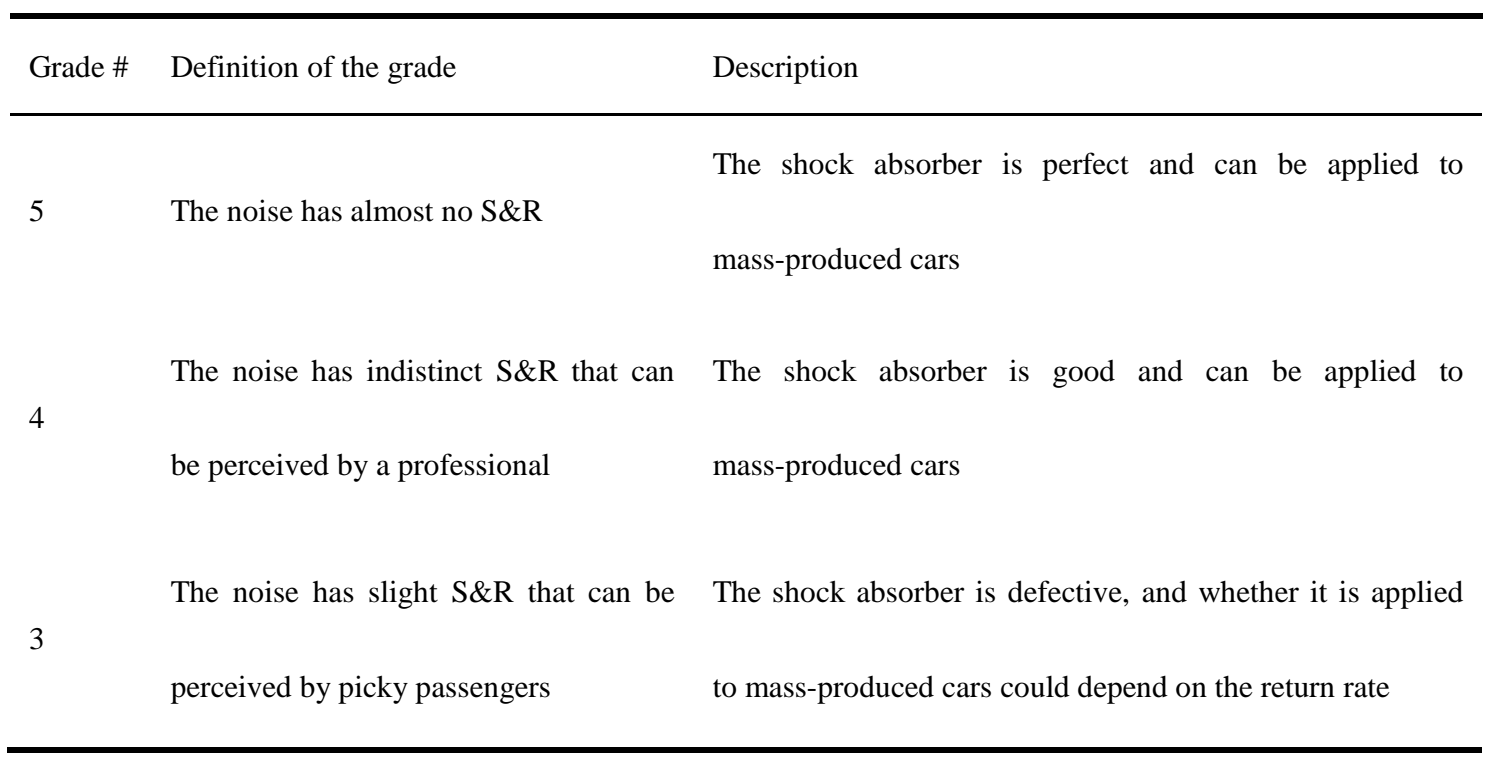


The noise has loud S\&R that can be The shock absorber is bad and should not be applied to 2
perceived by general passengers
mass-produced cars

The noise has serious S\&R that can be The shock absorber is terrible and cannot be applied to

1

perceived by all passengers mass-produced cars

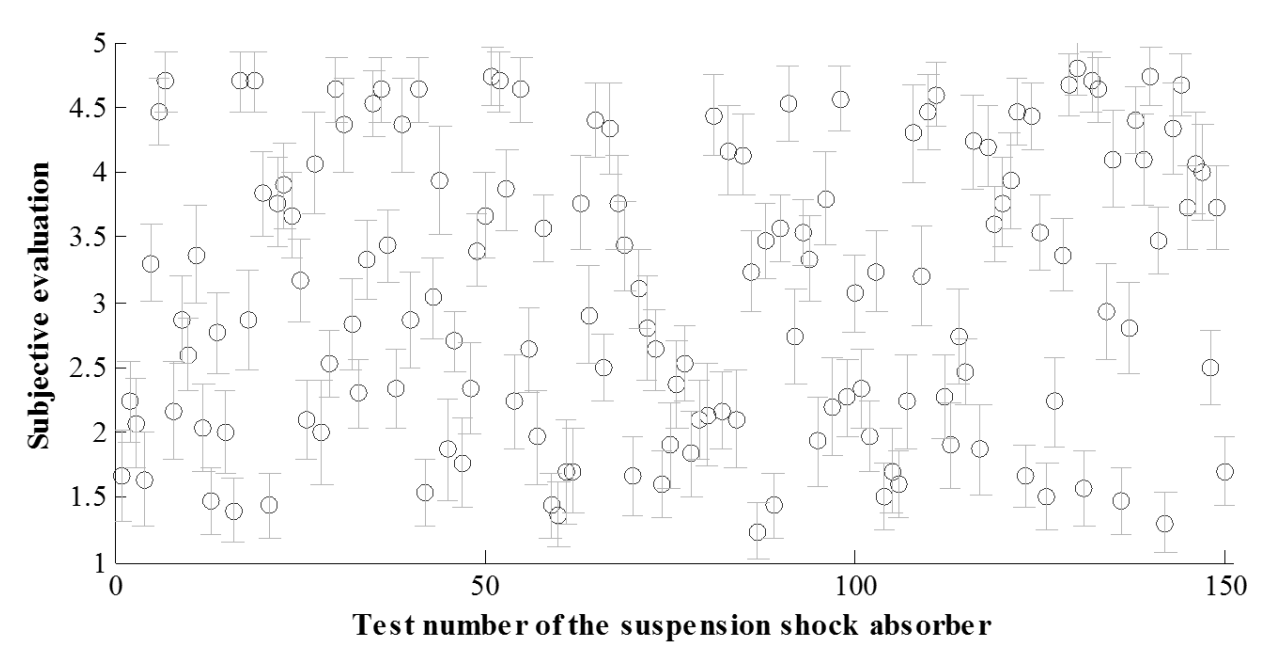

Fig. 6. Subjective rating of the S\&R of 150 suspension shock absorber noises, where the open circles ("o") are the average subjective scores and the vertical bars ("I") are the error bars corresponding to the standard deviation for each subjective evaluation.

\subsection{Test rig for suspension shock absorber S\&R noise}

The shock absorber samples, which had been tested in the vehicular road test, were also used for experimental analysis in a test rig. Special fixtures were made to imitate the joint form of the shock absorber by considering the assembly mode, in which shock absorbers in real vehicles are connected to the suspension and car body. The MTS 850 shock absorber test rig was used in the test, and each of the shock absorber samples was assembled to the test rig with customized fixtures, as shown in Fig. $\mathbf{7 .}$ Accelerometers were affixed to the top (piston rod) and the bottom (acting point) of the shock absorber to record the vibration signals. The sampling rate and length of the acquisition time were the same as in the vehicular road test. Before the road excitation signal was applied to the test rig, a linear averaging technique was introduced to reduce the noise interference and generate a more general loading signal, as described in Eq. 10. 


$$
x_{\text {rig }}(t)=\frac{1}{N} \sum_{i=1}^{N} x_{\text {road }}^{i}(t)
$$

where $x_{\text {road }}^{i}(t)$ is the recorded road excitation signal in the $i$ th vehicular road test, $N$ is the number of road excitation signals, and $x_{\text {rig }}(t)$ is the loading signal of the test rig. The post-processed excitation signal spectrum is shown in Fig. 8. As it can be observed in this figure, the excitation signal is primarily distributed at frequencies below $100 \mathrm{~Hz}$ and is concentrated at a frequency of approximately $20 \mathrm{~Hz}$.

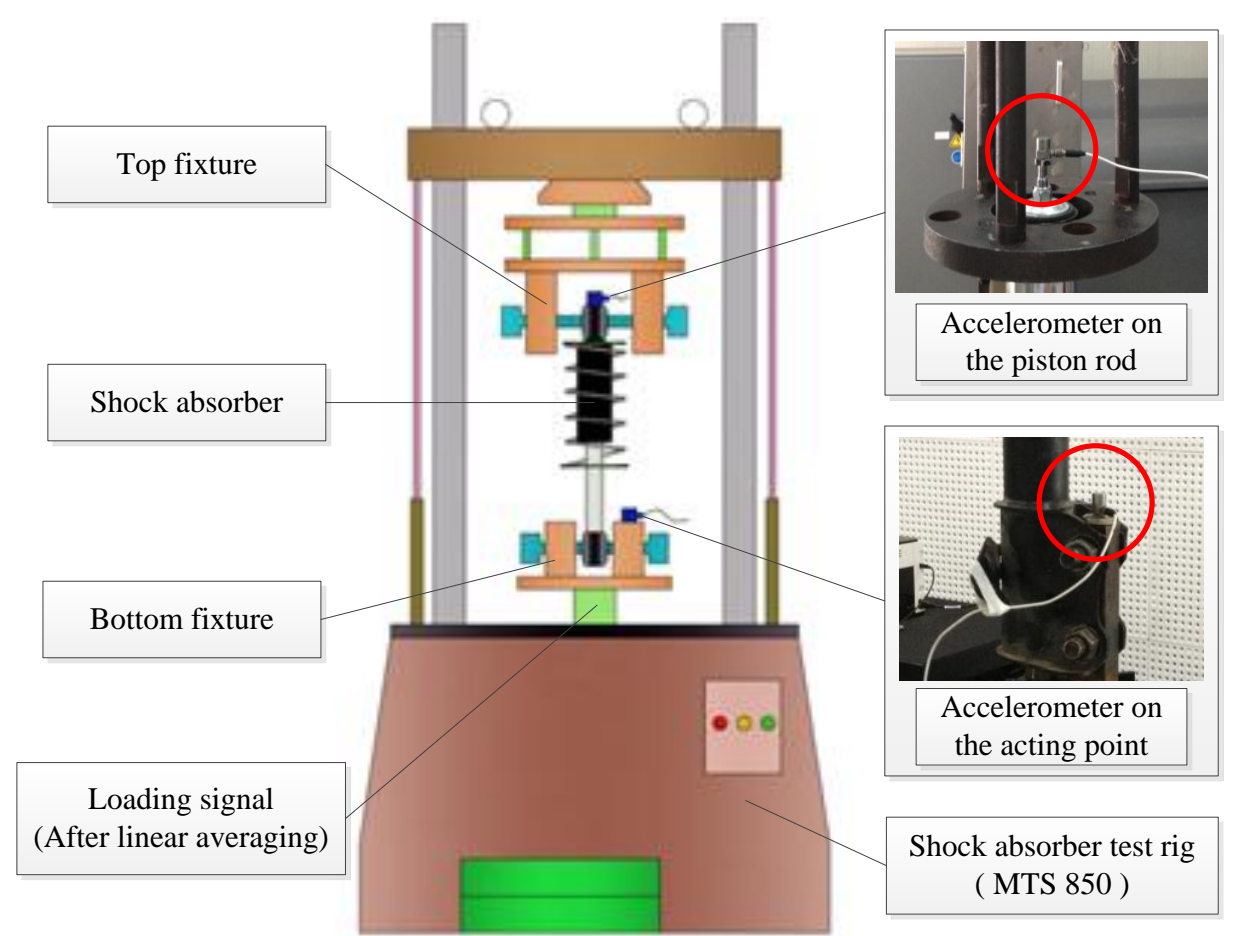

Fig. 7. Test rig for the identification of suspension shock absorber S\&R noise 


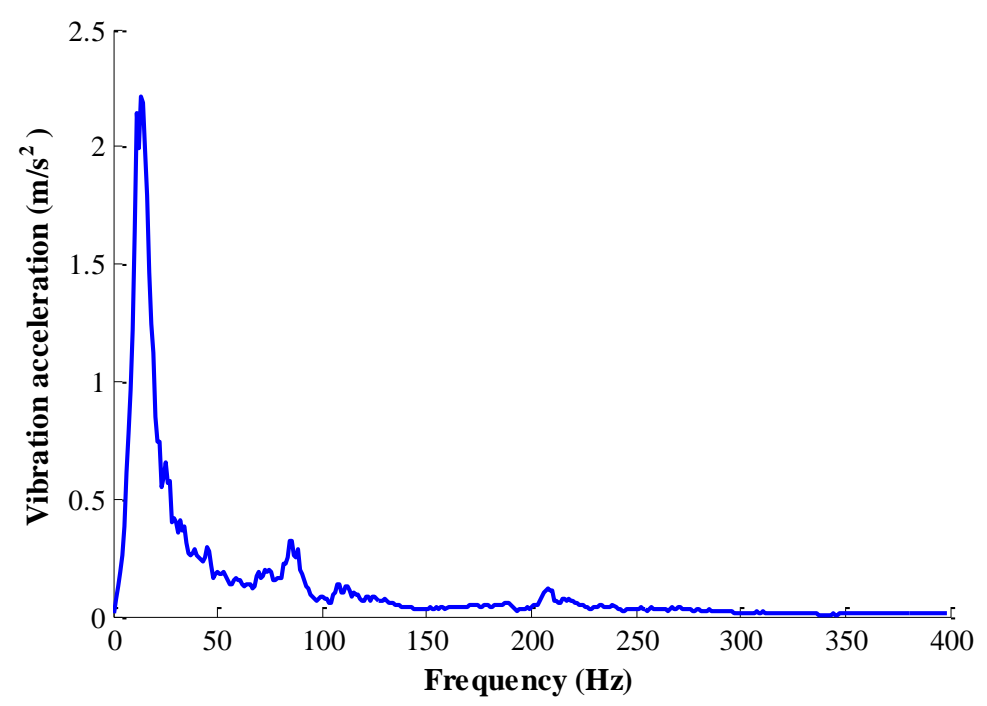

Fig. 8. Average excitation for the test rig

\subsection{Comparison of the road test and test rigs}

The vibration accelerations of the suspension shock absorber piston rod were measured in the vehicular road test, and some typical spectra of these vibration signals, which were distributed across the different grades of the shock absorber S\&R noise levels for subjective evaluation, are presented in Fig. 9 (a). This figure reveals that the $S \& R$ noise is proportional to the vibration characteristics of the shock absorber piston rod. Specifically, higher shock absorber vibration acceleration results in more serious interior S\&R noise, especially in the low-middle frequency band. Other recorded interior S\&R noises and piston rod vibration signals follow a similar trend. Therefore, the suspension shock absorber $\mathrm{S} \& \mathrm{R}$ noise can be reasonably represented by the vibration characteristics of the shock absorber piston rod, so we can simply use the vibration signal to identify the shock absorber S\&R noise. This observation presents a quantification method for measuring the $S \& R$ noise, so a relatively simple test rig can be used as a substitution for a complex vehicular road test to identify shock absorber S\&R noise.

Fig. 9 (b) shows the vibration spectra of the shock absorber piston rods that were measured in the test rig, which were the same samples as in Fig. 9 (a). Comparing Fig. 9 (a) to Fig. 9 (b) shows that the $S \& R$ pattern of the vehicular road test is highly consistent with that of the test rig. The primary difference in the piston rod vibration spectra between the road test and the rig test is the curving 
tendency at frequencies below $80 \mathrm{~Hz}$, which may have occurred because the rig test was a simplification of the vehicular road test and lacked some components of the vehicle's sub-systems, such as bushings, the suspension frame, and the car body. However, the shock absorber S\&R feature seems more obvious in the rig test than in the vehicular road test. Therefore, the piston rod vibration signal from the rig test is highly suitable for identifying suspension shock absorber S\&R noise.

Although the vibration spectra of the piston rod can, to some extent, reflect the grades of the suspension shock absorber $S \& R$ noise, these features are not sufficient to precisely identify such abnormal sound patterns using only this simple index. More accurate features are required to represent the characteristics of $\mathrm{S} \& \mathrm{R}$ noise. Therefore, an advanced signal processing method and an effective feature extraction technique should be introduced.

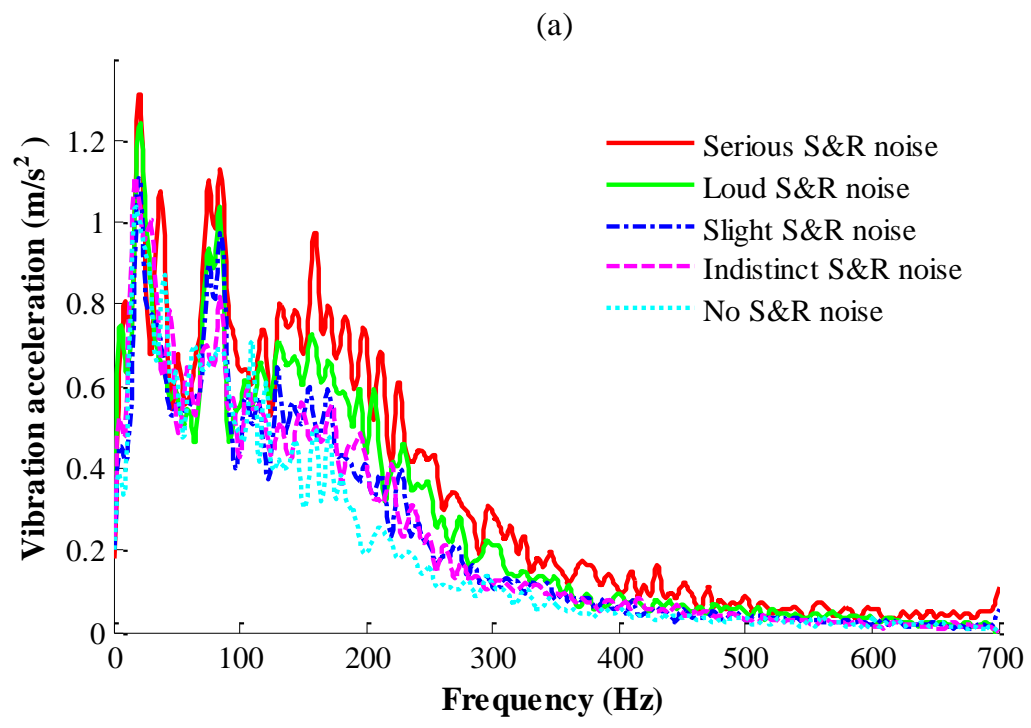

(b)

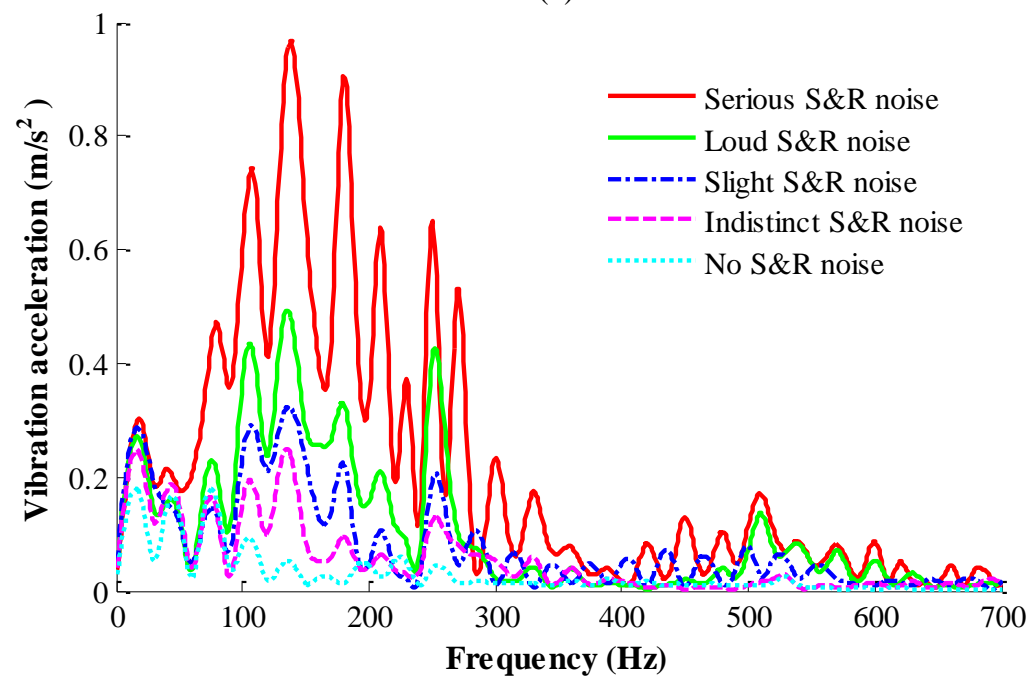


Fig. 9. Vibration spectra of a typical shock absorber piston rod, which depict different S\&R noise grades: (a) vehicular road test; (b) shock absorber rig test.

\section{Feature extraction for shock absorber S\&R noise}

Feature extraction is the foundation of pattern recognition. According to the experimental analysis in Section 3, some specific frequency components were generated in the piston rod vibration signals when shock absorber S\&R noise occurred. Therefore, piston rod vibration signals should be studied via the perspective of time-frequency analysis. Because the vibration signal was nonstationary and the $\mathrm{S} \& \mathrm{R}$ feature was distributed in a broadband range, we propose the WPT as our time-frequency processing method for the following two reasons. First, the WPT provides better control of the time and frequency resolution and offers more features of the original signal than the STFT and WT. Second, the WPT can decompose the signal to stable pre-defined frequency sub-bands, in which the EMD and its derivation algorithms are limited. Additionally, the decomposed signals should be represented by indices to numerically extract the S\&R features. The energy [32] and entropy [33] are two criteria that are often introduced in pattern identification issues. An energy feature describes a signal with physical significance, while an entropy feature describes a signal with respect to information theory, and they are applied in this study.

\subsection{WPT and energy based feature extraction}

The energy of the S\&R characteristics in a vibration signal change with the frequency distribution because of the nonlinearity of the suspension shock absorber system. Therefore, the wavelet packet energy (WPE) was proposed in this work. If the analysis level for the WPT is small, the energy features will be rough and cannot effectively represent the $S \& R$ characteristics. However, if the analysis level is large, the energy features would contain many redundancy and thus increase the computational cost and affect the identification accuracy. An analysis level of 4 was adopted based on extensive experiments, and the parameter settings for the WPT are summarized in Tab. 2. The energy feature $E_{i}$ is the sum of the square of the wavelet packet coefficients and is defined in Eq. 11. 


$$
E_{i}=\int_{-\infty}^{+\infty}\left|c_{i}(t)\right|^{2} d t \stackrel{t \in \text { finite }}{=} \sum_{t}\left[c_{i}(t)\right]^{2}, i=1,2, \ldots, 16
$$

where $c_{i}(t)$ is the wavelet packet coefficient, which represents the approximate coefficient or the detail coefficient. According to the conservation of energy and the orthogonality of the WPT decomposition, the sum of the WPE should be equal to the total energy of the original signal. A normalization technique was applied for further analysis, and a new feature vector was constructed as follows:

$$
W P E=\left[E_{1} / E, E_{2} / E, \ldots, E_{16} / E\right], \quad E=\left(\sum_{i=1}^{16}\left|E_{i}\right|^{2}\right)^{\frac{1}{2}}
$$

where $E$ is the normalization factor. Thus, the normalized vector, WPE, was obtained, which included 16 features for each individual signal.

Tab. 2 Parameter settings for the WPT analysis

\begin{tabular}{lll}
\hline Parameter type & Settings & Description \\
\hline Wavelet function & Daubechies & "db5" \\
Analysis levels & 4 & Denoising in $2^{4}=16$ sub-frequency band \\
Threshold determination & Soft threshold & Threshold $=2 \log (\operatorname{length}(x))$, where $x$ is the original signal \\
\hline
\end{tabular}

4.2 WPT and entropy based feature extraction

To some extent, suspension shock absorber $S \& R$ noise is a new component that is added to the normal vehicle interior noise, and this new information makes a difference in subjective evaluation. Entropy is commonly understood as a measure of disorder, with higher values of entropy always related to less system order and more contained information. Among many types of entropies, the sample entropy [34] is used extensively to quantify the complexity of a time series of data; therefore, it can be used to assess new information that the original signal contained. Compared to conventional approximate entropy [35], the sample entropy is less sensitive to noise and is more robust for short-length time-series signals because it does not include self-similar patterns [33]. In this subsection, the sample entropy is investigated as an index for suspension shock absorber S\&R feature extraction, and then the wavelet packet sample entropy (WPSE) is proposed. 
The sample entropy can be calculated via the following formula [36]:

$$
S E(m, r, N)=\lim _{N \rightarrow \infty}\left[-\ln \frac{B^{m+1}(r)}{B^{m}(r)}\right] \underset{N \in \text { finite }}{=}-\ln \frac{B^{m+1}(r)}{B^{m}(r)}
$$

where $m$ is the embedding dimension, $r$ is tolerance, $N$ is the number of data, $B^{m}(r)$ and $B^{m+1}(r)$ are distance related items. The sample entropy commonly sets parameter $m$ to be equal to 2 and the tolerance $r$ to be $r=0.2 * s t d$, where $s t d$ is the standard deviation of the time series data, because of the good statistical properties of these values. The detail calculation process for sample entropy is illustrated in [36].

Similar to the WPE, the WPSE is also determined using the wavelet packet coefficients of the piston rod vibration signal, and the WPT parameters are selected as in Tab. 2. Subsequently, the 16 decomposed wavelet packet coefficients are processed via Eq. (13), and the sample entropy vector is obtained as $\left[S E_{1}, S E_{2}, \ldots, S E_{16}\right]$. Then, the feature vector is normalized as

$$
W P S E=\left[S E_{1} / S E, S E_{2} / S E, \ldots, S E_{16} / S E\right], S E=\left(\sum_{i=1}^{16}\left|S E_{i}\right|^{2}\right)^{\frac{1}{2}}
$$

where $S E$ is the normalization factor.

\subsection{Comparison of the extracted features}

Tab. 3 presents the WPE of the piston rod vibration signals for the 5 typical S\&R noise grades. The WPE values of each specific S\&R grade are evidently different in most of the sub-bands, and the energy features of the S\&R characteristics are mainly distributed in the low-middle frequency, which is consistent with the experimental results in section 3. Objectively speaking, the WPE decreased in most of the wavelet packet coefficients when the grade of the S\&R noise gradually decreased. Tab. 4 shows the WPSE of the piston rod vibration signals for the 5 typical S\&R noise grades. A similar pattern was observed when comparing the WPSE to the WPE. The extracted features present a step-wise characteristic with gradually increasing S\&R levels. However, the difference is that the WPSE shows differences in both low-middle frequency bands and relatively high frequency bands. This observation means that the WPSE could extract some latent features from the original signals that the spectra and the WPE may not contain. However, the effectiveness of these extracted features must be further 
validated in the identification of suspension shock absorber $\mathrm{S} \& \mathrm{R}$ noise, which is discussed in more detail in section 5 .

Tab. 3 Normalized WPE values of the WPT sub-frequency band

\begin{tabular}{|c|c|c|c|c|c|c|c|c|}
\hline \multirow{2}{*}{$\mathrm{S} \& \mathrm{R}$ grades } & \multicolumn{8}{|c|}{ WPE values } \\
\hline & $\mathrm{WPE}_{1}$ & $\mathrm{WPE}_{2}$ & $\mathrm{WPE}_{3}$ & $\mathrm{WPE}_{4}$ & $\mathrm{WPE}_{5}$ & $\mathrm{WPE}_{6}$ & $\mathrm{WPE}_{7}$ & $\mathrm{WPE}_{8}$ \\
\hline Serious S\&R & 0.3790 & 0.7593 & 0.0812 & 0.6240 & 0.0144 & 0.0125 & 0.0584 & 0.0164 \\
\hline Loud S\&R & 0.3138 & 0.7255 & 0.0674 & 0.5238 & 0.0053 & 0.0119 & 0.0343 & 0.0129 \\
\hline Slight S\&R & 0.2634 & 0.7283 & 0.0208 & 0.5118 & 0.0035 & 0.0091 & 0.0168 & 0.0182 \\
\hline Indistinct $S \& R$ & 0.2011 & 0.6237 & 0.0133 & 0.3087 & 0.0026 & 0.0075 & 0.0179 & 0.0179 \\
\hline \multirow[t]{2}{*}{ Normal sound } & 0.1546 & 0.4966 & 0.0118 & 0.2334 & 0.0015 & 0.0048 & 0.0163 & 0.0153 \\
\hline & $\mathrm{WPE}_{9}$ & $\mathrm{WPE}_{10}$ & $\mathrm{WPE}_{11}$ & $\mathrm{WPE}_{12}$ & $\mathrm{WPE}_{13}$ & $\mathrm{WPE}_{14}$ & $\mathrm{WPE}_{15}$ & $\mathrm{WPE}_{16}$ \\
\hline Serious S\&R & 0.0003 & 0.0004 & 0.0026 & 0.0013 & 0.0098 & 0.0065 & 0.0024 & 0.0027 \\
\hline Loud S\&R & 0.0001 & 0.0003 & 0.0052 & 0.0006 & 0.0087 & 0.0055 & 0.0039 & 0.0036 \\
\hline Slight S\&R & 0.0001 & 0.0003 & 0.0021 & 0.0005 & 0.0124 & 0.0018 & 0.0021 & 0.0041 \\
\hline Indistinct $S \& R$ & 0.0003 & 0.0002 & 0.0026 & 0.0003 & 0.0104 & 0.0036 & 0.0035 & 0.0026 \\
\hline Normal sound & 0.0002 & 0.0001 & 0.0019 & 0.0002 & 0.0073 & 0.0038 & 0.0011 & 0.0025 \\
\hline
\end{tabular}

* The sub-frequency bands for $\mathrm{WPE}_{1}, \mathrm{WPE}_{2}, \ldots, \mathrm{WPE}_{16}$ are $0 \sim 80 \mathrm{~Hz}, 80 \sim 160 \mathrm{~Hz}, \ldots, 1200 \sim 1280 \mathrm{~Hz}$, respectively.

Tab. 4 Normalized WPSE values of the WPT sub-frequency band

\begin{tabular}{|c|c|c|c|c|c|c|c|c|}
\hline \multirow{2}{*}{ S\&R grades } & \multicolumn{8}{|c|}{ WPSE values } \\
\hline & $\mathrm{WPSE}_{1}$ & $\mathrm{WPSE}_{2}$ & $\mathrm{WPSE}_{3}$ & $\mathrm{WPSE}_{4}$ & WPSE5 & WPSE$_{6}$ & $\mathrm{WPSE}_{7}$ & WPSE8 \\
\hline Serious S\&R & 0.1017 & 0.2619 & 0.2155 & 0.2713 & 0.3080 & 0.3027 & 0.2188 & 0.2370 \\
\hline Loud S\&R & 0.0683 & 0.1877 & 0.1904 & 0.2361 & 0.2326 & 0.2470 & 0.1984 & 0.2210 \\
\hline Slight S\&R & 0.0442 & 0.1568 & 0.1780 & 0.2114 & 0.2416 & 0.2261 & 0.1961 & 0.1794 \\
\hline Indistinct S\&R & 0.0472 & 0.1396 & 0.1591 & 0.2025 & 0.2068 & 0.2161 & 0.1671 & 0.1304 \\
\hline Normal sound & 0.0397 & 0.1093 & 0.1234 & 0.1269 & 0.1433 & 0.1720 & 0.1391 & 0.1289 \\
\hline
\end{tabular}




\begin{tabular}{lccccccccc}
\hline & WPSE & WPSE $_{10}$ & WPSE $_{11}$ & WPSE $_{12}$ & WPSE $_{13}$ & WPSE $_{14}$ & WPSE $_{15}$ & WPSE $_{16}$ \\
\cline { 2 - 8 } Serious S\&R & 0.3036 & 0.3373 & 0.3500 & 0.3764 & 0.3442 & 0.2934 & 0.3499 & 0.2571 \\
Loud S\&R & 0.2845 & 0.3112 & 0.3231 & 0.3360 & 0.3003 & 0.2606 & 0.3159 & 0.2947 \\
Slight S\&R & 0.2351 & 0.3182 & 0.3004 & 0.3179 & 0.2762 & 0.2528 & 0.2994 & 0.2912 \\
Indistinct S\&R & 0.2134 & 0.3087 & 0.2876 & 0.3048 & 0.2427 & 0.2210 & 0.2444 & 0.2268 \\
Normal sound & 0.1624 & 0.2841 & 0.2604 & 0.2771 & 0.2380 & 0.1960 & 0.2018 & 0.2763 \\
\hline
\end{tabular}

* The sub-frequency bands for WPSE $\mathrm{WPSE}_{2}, \ldots, \mathrm{WPSE}_{16}$ are $0 \sim 80 \mathrm{~Hz}, 80 \sim 160 \mathrm{~Hz}, \ldots, 1200 \sim 1280 \mathrm{~Hz}$, respectively.

\section{GA-SVM modeling for S\&R identification and comparison}

This section describes the identification of suspension shock absorber S\&R noise based on the extracted features from Section 4 and the SVM model. Here we compare the GA and the grid search (GS) methods to optimize the hyper-parameters of SVM, and furthermore introduce another intelligent model to verify the identification capability of our developed model. All the computational experiments were implemented in MATLAB 2016a on a $2.40 \mathrm{GHz}$ 4-core 8-thread Intel Core i7-4700MQ CPU mobile workstation with $16 \mathrm{~GB}$ of memory. We conducted the computational experiments 50 times to account for the stochastic characteristics of the intelligent algorithms, and the average performances are reported in the following study.

\subsection{GA-SVM modeling and parameter optimization}

The RBF, whose form is shown in Eq. (9), was applied as the kernel function of the SVM to set up the GA-SVM model because of its good generalization performance and few parameters to be turned. The soft margin parameter $C$ and the kernel parameter $\gamma$ should be carefully optimized for this RBF-based SVM. The search ranges for the parameters $C$ and $\gamma$ are selected as $0.01 \sim 1000$ and $0.1 \sim$ 100, respectively, and parameter settings for GA are summarized in Tab. 5. The dataset of shock absorber piston rod vibration signals consists of 150 samples with 16 features each (for both WPE and WPSE), and we randomly split the dataset to a training set, a validation set and a testing set, which contained 70, 40, and 40 samples, respectively. These two features were used separately as the input feature to compare the effectiveness of the WPE and WPSE, and the corresponding S\&R noise grade 
was exported as the output target. Meanwhile, the best combination of the feature subsets could be attained through the GA-SVM model. The procedure for developing the GA-SVM is shown in Fig. 4.

Tab. 6 presents the final feature subset selection results, and Fig. 10 shows the change in the fitness function values with an increasing number of iterations in one experiment. Tab. 6 indicates that nearly half of the WPE features (9 out of 16) were selected as useful features, and almost all were in the low-middle frequency band. The remaining features for the WPSE, except for the 16th sub-band (15 out of 16), demonstrated their effectiveness in the identification of suspension shock absorber S\&R noise. These results mainly occurred because most of the energy in a signal lies in low-middle frequencies, with less in high frequencies; therefore, the energy features lack sensitivity in extracting characteristics in the high-frequency band. However, the sample entropy extracts features from the perspective of information complexity, so it can measure the amount of new information that any frequency band contained. Fig. 10 clearly illustrates that applying the WPSE as the input feature shows a better capability than the WPE when using the GA-SVM model; the WPSE-based GA-SVM model achieved a classification accuracy rate of $97.5 \%$ while the WPE based GA-SVM attained $95.0 \%$ in the validation set. The best combinations of parameters, namely, $\mathrm{C}=5.61$ and $\gamma=0.78$ for the WPSE-based GA-SVM and C $=77.62$ and $\gamma=0.39$ for the WPE-based GA-SVM, were obtained through GA optimization. Moreover, the traditional GS method for selecting SVM parameters was conducted to show the superiority of the GA optimization approach. The search grids for the parameters $C$ and $\gamma$ are set as $\left\{2^{-5}, 2^{-4}, \ldots, 2^{9}, 2^{10}\right\}$ and $\left\{2^{-3}, 2^{-2}, \ldots, 2^{6}, 2^{7}\right\}$, respectively, and the final selected WPE and WPSE features, which are shown in Tab. 6, were used as the GS-SVM model input feature. The comparison results are summarized in Tab. 7; for the same input feature, the GA-optimized SVM improved the average classification accuracy rate by an absolute percentage of at least 3\% compared to the GS-optimized SVM.

Tab. 5 Parameter settings for the GA process

\begin{tabular}{lll}
\hline Parameter type & Settings & Description \\
\hline
\end{tabular}




\begin{tabular}{|c|c|c|}
\hline Chromosome length & & 12 bits for $C, 8$ bits for $\gamma$, and 1 bit for each feature \\
\hline (binary code) & 36 & subset selection \\
\hline Population size & 100 & $\begin{array}{l}\text { Number of chromosomes that is created in each } \\
\text { generation }\end{array}$ \\
\hline Crossover rate & 0.5 & Probability of crossover \\
\hline Mutation rate & 0.05 & Probability of mutation \\
\hline Number of generations & 200 & Max number of iterations \\
\hline Fitness function & Classification accuracy & Classification accuracy of the validation data \\
\hline
\end{tabular}

Tab. 6 Best combination of feature subsets. For each WPT sub-band feature, 1 means the corresponding feature has been selected for the model input, whereas 0 means it has not been selected.

\begin{tabular}{llllllllllllllllllll}
\hline \multicolumn{10}{c}{ Feature subset selection } \\
\hline Sub-band & 1 & 2 & 3 & 4 & 5 & 6 & 7 & 8 & 9 & 10 & 11 & 12 & 13 & 14 & 15 & 16 \\
WPE & 1 & 1 & 1 & 1 & 1 & 1 & 1 & 0 & 0 & 1 & 0 & 1 & 0 & 0 & 0 & 0 \\
WPSE & 1 & 1 & 1 & 1 & 1 & 1 & 1 & 1 & 1 & 1 & 1 & 1 & 1 & 1 & 1 & 0 \\
\hline
\end{tabular}




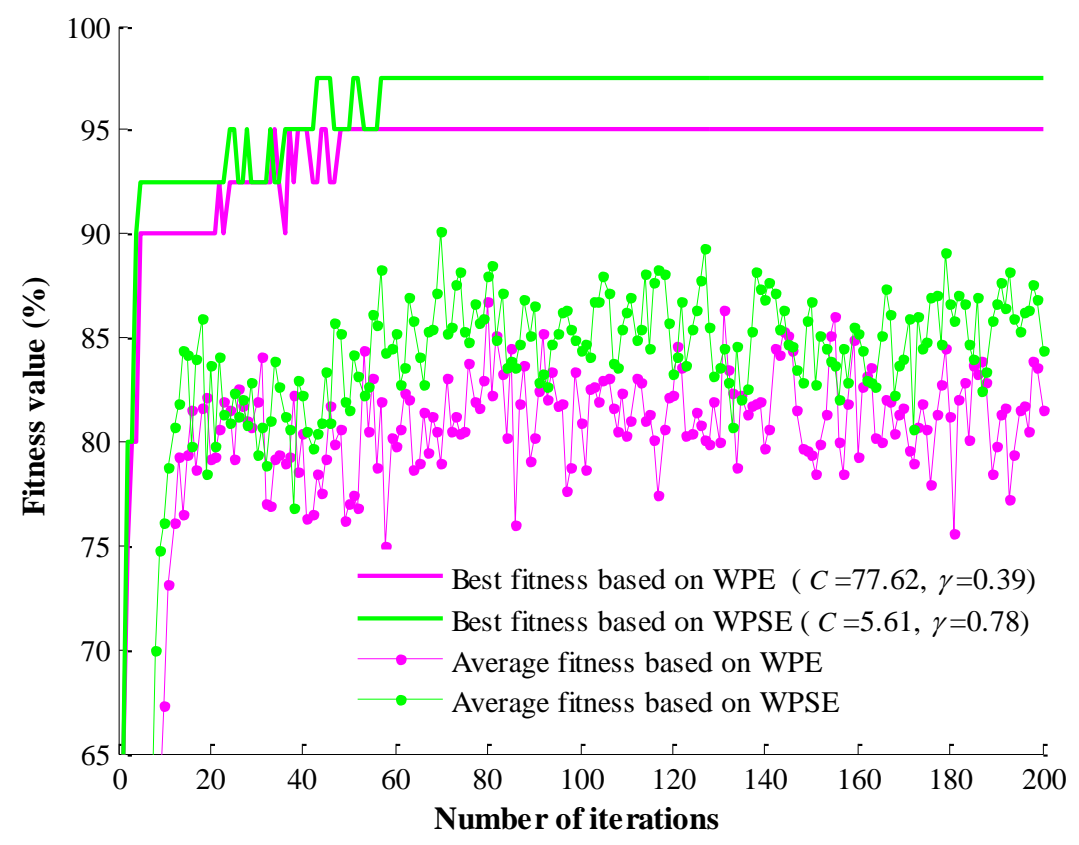

Fig. 10. Fitness function values of the GA-SVM model

Tab. 7 Performance comparison between the GA- and GS-optimized SVM

\begin{tabular}{|c|c|c|c|}
\hline \multirow{2}{*}{\multicolumn{2}{|c|}{ Classification accuracy (validation set) }} & \multicolumn{2}{|c|}{ Parameter optimization algorithm type } \\
\hline & & GA-SVM & GS-SVM $(C=64$ and $\gamma=2)$ \\
\hline & WPE & $97.33 \%$ & $93.28 \%$ \\
\hline \multicolumn{4}{|l|}{ Feature type } \\
\hline & WPSE & $94.76 \%$ & $91.62 \%$ \\
\hline
\end{tabular}

\subsection{S\&R identification and model comparison}

The filtered feature subsets and the parameter-optimized SVM model were obtained according to subsection 5.1. To verify the effectiveness and efficiency of the newly developed model, another state-of-the-art method, a genetic algorithm optimized back propagation neural network (GA-BPNN), was introduced in this study to evaluate the suspension shock absorber $S \& R$ noise.

Similar to the GA-SVM approach, the crucial parameters of BPNNs, including the weights between the contiguous layers and biases of a layer, should be encoded in a chromosome and optimized through the GA process. A BPNN with three layers, namely, one input layer, one hidden layer and one output layer, was applied. Following the guide of $[37,38]$ for model selection, the structure of the 
BPNN was set as 15-31-1, and the transfer function, training function and learning function were selected as "tansig", "trainlm" and "learngdm", respectively. The learning rate was set to 0.05 in each epoch, and the learning process was performed until either the number of iterations reached 1,000 or the classification accuracy rate reached $100 \%$. The filtered WPSE feature subsets were used as the model input, and the corresponding S\&R noise level was applied as the output. The development of the GA-BPNN and the comparison procedure between the GA-SVM and GA-BPNN are presented in Fig.

11.

Tab. 8 summarizes the performances of the two models. The results show that the average classification accuracy rate of the GA-SVM is $96.67 \%$ and that of the GA-BPNN is $95.24 \%$, which demonstrates the effectiveness of the GA-SVM method compared to the GA-BPNN method. Meanwhile, the average running time of the GA-BPNN was nearly three times that of the GA-SVM, which were $56.53 \mathrm{~s}$ and $19.68 \mathrm{~s}$, respectively. These results mainly occurred because the free parameters of the SVM model that needed to be optimized were much fewer than those of the BPNN model. Based on the above analysis, the GA-SVM model is superior in the identification of suspension shock absorber S\&R noise compared to the GA-BPNN model in effectiveness and efficiency and thus can be applied to solve complex practical problems effectively. 


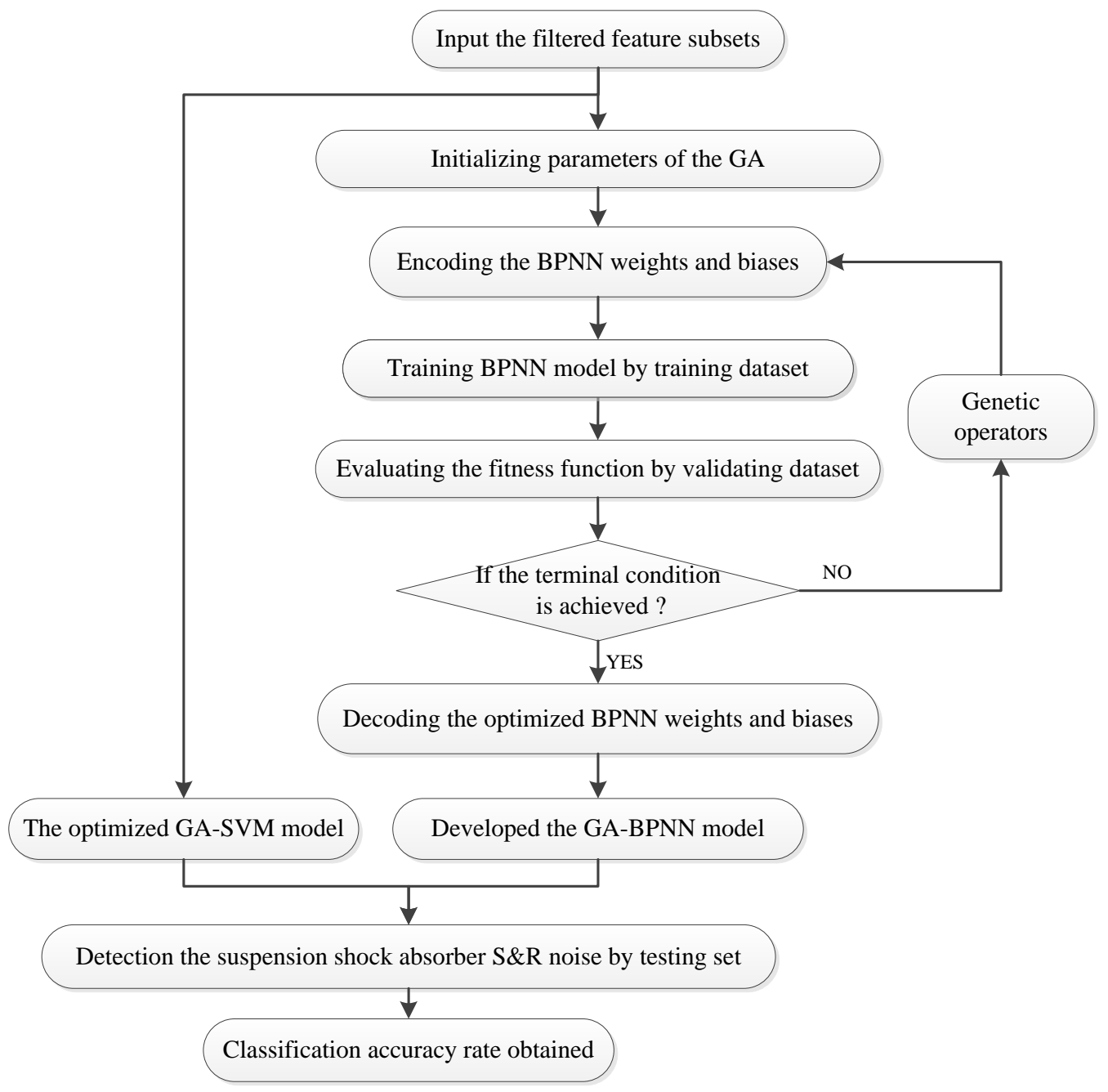

Fig. 11. Development process of GA-BPNN and the comparison between GA-SVM (obtained in section 5.1) and GA-BPNN.

Tab. 8 Identification performances of the GA-SVM and GA-BPNN models.

\begin{tabular}{lll}
\hline & GA-SVM & GA-BPNN \\
\hline Classification accuracy (testing set) & $96.47 \%$ & $95.24 \%$ \\
Running time & $19.68 \mathrm{~s}$ & $56.53 \mathrm{~s}$ \\
\hline
\end{tabular}

\section{Conclusions}

In this paper, an intelligent methodology that combines a wavelet packet transform (WPT) and genetic algorithm-optimized support vector machine (GA-SVM) was proposed for the identification of vehicular suspension shock absorber S\&R noise. According to a vehicular road test and shock absorber rig test, suspension shock absorber S\&R noise shows direct proportionality to the vibration characteristics of a shock absorber piston rod. Therefore, rig tests can be applied as a substitution to 
identify shock absorber S\&R noise. Two extracted features, namely, wavelet packet energy (WPE) and wavelet packet sample entropy (WPSE), were used and compared to each other, which demonstrated that the WPSE could extract more useful information regarding S\&R characteristics in the original signal than the WPE. Effective feature subsets and optimized SVM model parameters could be obtained through the genetic algorithm, which forms the GA-SVM approach. In addition, a GA-BPNN model was applied to verify the performance of the newly proposed model. The experiments showed that the GA-SVM model (96.47\% classification accuracy and $19.68 \mathrm{~s}$ running time) was better than the GA-BPNN model (95.24\% classification accuracy and $56.53 \mathrm{~s}$ running time) in effectiveness and efficiency. As an intelligent method, retraining and extending the GA-SVM might be regarded as an effective and promising technique for fault identification in other engineering fields.

\section{Acknowledgments}

This work was supported by the Chinese National Science Foundation Grant (No. 51475387) and the Key Science and Technology Research Program in Sichuan Province, China (No. 2015GZ0126). Special thanks to the China Automotive Technology \& Research Center for their kind supply of the research conditions.

\section{References}

[1] Pietila G, Lim TC. Intelligent systems approaches to product sound quality evaluations - a review. Appl Acoust 2012;73:987-1002. doi: 10.1016/j.apacoust.2012.04.012.

[2] Lee S, Kim TG, Lim JT. Characterization of an axle-gear whine sound in a sports utility vehicle and its objective evaluation based on synthetic sound technology and an artificial neural network. Proc Inst Mech Eng, Part D: J Automob Eng 2008;222:383-96. doi: 10.1243/09544070JAUTO563. 
[3] Yoon J, Yang I, Jeong J, Park S, Oh J. Reliability improvement of a sound quality index for a vehicle HVAC system using a regression and neural network model. Appl Acoust 2012;73:1099-103. doi: 10.1016/j.apacoust.2012.02.018.

[4] Xu ZM, Zhang Y, He YS, Zhang ZF. Subjective and objective analysis on the sound quality of automotive wiper system. J Shock Vib 2013;36:14-7.

[5] Huang HB, Li RX, Yang Q, Ding WP, Yang ML. Rig test for identifying abnormal noise of suspension shock absorber. J Vib Shock 2015;34:191-6.

[6] Benaziz M, Nacivet S, Thouverez F. A shock absorber model for structure-borne noise analyses. J Sound Vib 2015;349:177-94. doi: 10.1016/j.jsv.2015.03.034.

[7] Roger J, Johan O, Matti R. A new test track for automotive squeak and rattle detection. Appl Acoust 2014;80:79-85.

[8] Song R, Ding WP, Yang ML, Li Z, Xu XC. Experimental research on structure transfer sound of automobile suspension shock absorber. Automob Technol 2011;7:39-42.

[9] Benaziz M, Nacivet S, Deak J, Thouverez F. Double tube shock absorber model for noise and vibration analysis. SAE Int J Passeng Cars - Mech Syst 2013;6:1177-85. doi: 10.4271/2013-01-1912.

[10] Yao MT, Guan JF, Gu L. Study on abnormal noise of vehicle twin-tube shock absorber. Mach Design Manuf 2011;2:114-6.

[11] Zhang LJ, Yu ZP, Jin XX. Experimental research on the abnormal moise of hudraulic suspension shock absorber. J Vib Shock 2002;21:33-8.

[12] Huang HB, Li RX, Huang XR, Yang ML, Ding WP. Sound quality evaluation of vehicle suspension shock absorber rattling noise based on the Wigner-Ville distribution. Appl Acoust 2015;100:18-25. doi: 10.1016/j.apacoust.2015.06.018.

[13] Shu H, Luo S, Wang L. Test method, simulation and micro-process dynamic model for noise analysis of auto hydraulic shock absorber. SAE Technical Paper No. 2015-01-2351; 2015. doi: 10.4271/2015-01-2351. 
[14] Heo Y, Kim K. Definitions of non-stationary vibration power for time-frequency analysis and computational algorithms based upon harmonic wavelet transform. J Sound Vib 2015;336:275-92. doi: 10.1016/j.jsv.2014.10.025.

[15] Torres R, Torres E. Fractional Fourier analysis of random signals and the notion of/spl alpha/-Stationarity of the Wigner-Ville distribution. IEEE Trans Signal Process 2013;61:1555-60. doi: 10.1109/TSP.2012.2236834.

[16] Guo W, Tse PW. A novel signal compression method based on optimal ensemble empirical mode decomposition for bearing vibration signals. J Sound Vib 2013;332:423-41. doi: 10.1016/j.jsv.2012.08.017.

[17] Lorenz C, Ferraudo AS, Suesdek L. Artificial Neural Network applied as a methodology of mosquito species identification Acta Trop 2015;152:165-9. doi: 10.1016/j.actatropica.2015.09.011.

[18] Kaytez F, Taplamacioglu MC, Cam E, Hardalac F. Forecasting electricity consumption: a comparison of regression analysis, neural networks and least squares support vector machines. Int J Electr Power Energ Syst 2015;67:431-8. doi: 10.1016/j.ijepes.2014.12.036.

[19] Atak O, Huybrechs D, Pluymers B, Desmet W. The design of Helmholtz resonator based acoustic lenses by using the symmetric Multi-Level Wave Based Method and genetic algorithms. J Sound Vib 2014;333:3367-81. doi: 10.1016/j.jsv.2014.03.033.

[20] El-Bakari A, Khamlichi A, Jacquelin E, Dkiouak R. Assessing impact force localization by using a particle swarm optimization algorithm. J Sound Vib 2014;333:1554-61. doi: 10.1016/j.jsv.2013.11.032.

[21] Wang YS, Lee C, Kim DG, Xu Y. Sound-quality prediction for nonstationary vehicle interior noise based on wavelet pre-processing neural network model. J Sound Vib 2007;299:933-47. doi: 10.1016/j.jsv.2006.07.034. 
[22] Wang Y, Xu G, Liang L, Jiang K. Detection of weak transient signals based on wavelet packet transform and manifold learning for rolling element bearing fault diagnosis. Mech Syst Signal Process 2015;54-55:259-76. doi: 10.1016/j.ymssp.2014.09.002.

[23] Vapnik V. The nature of statistical learning theory. Berlin: Springer Verlag Science; 1995.

[24] Choi S. Detection of valvular heart disorders using wavelet packet decomposition and support vector machine. Expert Syst Appl 2008;35:1679-87. doi: 10.1016/j.eswa.2007.08.078.

[25] Orrù G, Pettersson-Yeo W, Marquand AF, Sartori G, Mechelli A. Using support vector machine to identify imaging biomarkers of neurological and psychiatric disease: a critical review. Neurosci Biobehav Rev 2012;36:1140-52. doi: 10.1016/j.neubiorev.2012.01.004.

[26] Liu H, Zhang J, Guo P, Bi F, Yu H, Ni G. Sound quality prediction for engine-radiated noise. Mech Syst Signal Process 2015;56-57:277-87. doi: 10.1016/j.ymssp.2014.10.005.

[27] Huang C. A hybrid stock selection model using genetic algorithms and support vector regression. Appl Soft Comput 2012;12:807-18. doi: 10.1016/j.asoc.2011.10.009.

[28] GB/T 18697-2002: Acoustics — method for measuring vehicle interior noise. 2002.

[29] Liu X, Wang H, Shan Y, He T. Construction of road roughness in left and right wheel paths based on PSD and coherence function. Mech Syst Signal Process 2015;60-61:668-77. doi: 10.1016/j.ymssp.2015.01.034.

[30] Lee S. Objective evaluation of interior sound quality in passenger cars during acceleration. $\mathbf{J}$ Sound Vib 2008;310:149-68. doi: 10.1016/j.jsv.2007.07.073.

[31] Wang YS, Shen GQ, Xing YF. A sound quality model for objective synthesis evaluation of vehicle interior noise based on artificial neural network. Mech Syst Signal Process 2014;45:255-66. doi: 10.1016/j.ymssp.2013.11.001.

[32] Zhao X, Hu Z, Li R, Zhou C, Jiang J. Internal leakage fault feature extraction of hydraulic cylinder using wavelet packet energy. In: Li K, Xiao Z, Wang Y, Du J, Li K, editors. Parallel computational fluid dynamics, Berlin: Springer Verlag; 2014, p. 363-375. 
[33] Boškoski P, Juričić Đ. Fault detection of mechanical drives under variable operating conditions based on wavelet packet Rényi entropy signatures. Mech Syst Signal Process 2012;31:369-81. doi: 10.1016/j.ymssp.2012.04.016.

[34] Widodo A, Shim M, Caesarendra W, Yang B. Intelligent prognostics for battery health monitoring based on sample entropy. Expert Syst Appl 2011;38:11763-9. doi: 10.1016/j.eswa.2011.03.063.

[35] Richman JS, Moorman JR. Physiological time-series analysis using approximate entropy and sample entropy. Am J Physiol Heart Circ Physiol 2000;278:H2039-49.

[36] Pincus SM. Assessing serial irregularity and its implications for health. Ann N Y Acad Sci 2001;954:245-67. doi: 10.1111/j.1749-6632.2001.tb02755.x.

[37] Laudani A, Lozito GM, Riganti Fulginei F, Salvini A. On training efficiency and computational costs of a feed forward neural network: a review. Comput Intell Neurosci 2015;2015:818243. doi: $10.1155 / 2015 / 818243$.

[38] Xing YF, Wang YS, Shi L, Guo H, Chen H. Sound quality recognition using optimal wavelet-packet transform and artificial neural network methods. Mech Syst Signal Process 2016;66-67:875-92. doi: 10.1016/j.ymssp.2015.05.003. 